\title{
DESARROLLO DE UN INSTRUMENTO PARA EVALUACIÓN DEL RESENTIMIENTO EN JÓVENES
}

\section{DEVELOPING AN INSTRUMENT TO ASSESS RESENTMENT IN YOUNG PEOPLE}

\author{
Francisco Leal-Soto \\ Johanna Sepúlveda Muñoz \\ Sara Vargas Gómez \\ Marcelo Braniff Carrasco \\ Mitchael Irrazábal Leiva \\ Universidad de Tarapacá, Chile
}

\begin{abstract}
Resumen: Se presentan dos estudios que informan del desarrollo y validación de un instrumento para evaluar resentimiento en jóvenes. En el primero participaron 60 jóvenes infractores de la ley, 30 considerados resentidos y 30 considerados no resentidos por profesionales con conocimiento de los jóvenes. Se desarrolló un instrumento de 74 ítems validados por expertos, el que se aplicó a los participantes. El análisis de ítems permitió reducir la escala a 36 ítems, con buena consistencia interna, que discriminan claramente entre jóvenes resentidos y no resentidos. En el segundo estudio, se reconfiguró la escala que quedó conformada por 40 ítems, y se aplicó a una muestra representativa de 575 estudiantes secundarios. El análisis factorial arrojó tres factores coherentes con la operacionalización del constructo, que se denominaron reacción ante la injusticia, afán vindicativo y valoración, aunque explican una proporción sólo moderada de la varianza. Se concluye que el instrumento resulta válido para evaluar resentimiento en jóvenes.
\end{abstract}

Palabras Clave: resentimiento; jóvenes; evaluación; validación

\begin{abstract}
Two studies about development and validation of an instrument to assess resentment in young people are presented. The first one included 60 juvenile offenders, half of whom were considered resentful and the other half were not considered resentful by experts that work with them. An instrument of 74 items validated by experts was developed and was applied to the participants. Item analyses were performed, resulting in a 36 item scale. This resulting scale had good reliability and clearly discriminated between resentful and not resentful young. In the second study, the scale was reconfigured, composed by 40 items and was applied to a representative sample of 575 high school students. Factor analysis of the results yielded 3 factors consistent with the operationalization of the construct: reaction to injustice, vindictive and appreciation, although the amount of explained variance is only moderate. It is concluded that the instrument is valid to assess resentment in young people.
\end{abstract}

Key Words: resentment; young people; assessment; validation

Del resentimiento se ha llegado a afirmar que es la más universal entre las emociones fútiles y destructivas que puede experimentar el ser humano (Dalrymple, 1995). En su uso habitual, resentimiento es un término que tiene un significado directo y evidente, y como tal, se lo utiliza frecuentemente como explicación de muchos fenómenos individuales y sociales, en particular desde la sociología (e. g., Barbalet, 2001, citado en Navarro, 2007). En el ámbito psicoterapéutico, el resentimiento también aparece, por ejemplo, en Fritz Perls (1969/1987), quien lo señala como la situación inconclusa por excelencia, ya que quienes están resentidos no pueden avanzar ni liberarse, ni expresar su enojo o modificar el mundo para obtener satisfacción, ni tampoco olvidar el asunto que les molesta. Feather y Sherman (2002) y Feather y Nairn (2005) han establecido que el resentimiento influye en las respuestas emocionales de las personas respecto de los triunfos y fracasos de otros, incluso más que la envidia. En un sentido positivo, Rollo May (1976) indica que la presencia de resentimiento es un elemento 
de pronóstico psicoterapéutico positivo, ya que sería indicador de fuerza interior para resistir a las presiones externas. Se ha descrito su importancia en situaciones críticas de injusticia social como el caso de perseguidos y refugiados (Rees \& Silove, 2011), y en ámbitos como la política, en que se lo ha identificado como importante factor de voto (Knuckey, 2011). Inga y Vara (2006) reportan que el resentimiento es la variable que mejor predice -negativamentela satisfacción de los ancianos con su vida, y Aggar, Ronaldson y Cameron (2011) encontraron que el resentimiento se relaciona con mayor ansiedad y depresión en los cuidadores de ancianos desvalidos. También se lo ha identificado como mediador de la recuperación del consumo de drogas en los abordajes basados en la fe (Lyons, Deane, Caputi \& Kelly, 2011), y como vehiculizador de prácticas, discursos y políticas racistas a través de los espacios afectivos y emocionales en la escuela (Zembylas, 2010).

Por el carácter principalmente moral que asume, el resentimiento ha sido tratado prioritariamente por filósofos. Nietzche (1887/1993) presenta el amor cristiano como el mejor ejemplo del resentimiento, al surgir desde la impotencia para manifestar el odio o realizar la venganza: al quedar vedada la posibilidad de actuar para expresar tales sentimientos por el mandato cristiano, éstos serían reprimidos dando lugar al resentimiento, el que, al ser sublimado, generaría el movimiento creador que culminaría en el amor cristiano. Independientemente de que estemos de acuerdo o no con el ejemplo de Nietzche, éste destaca uno de los elementos centrales presentes en el resentimiento: la imposibilidad de expresar activamente los sentimientos negativos y agresivos hacia otras personas. Coincidiendo con esto, May (1976) señala que el resentimiento es la apariencia del odio en la vida urbana y civilizada, y Delgado (1938) advertía que se trata de una hostilidad sin objeto individual exclusivo y en que la movilización no se actualiza de manera adecuada o directa, sino indirectamente, con transposiciones, agresividad difusa, envidia velada, rencor sordo. Scheler (1912/1938) lo describe como una reacción emocional recurrente, que se vive y revive repetidamente con una cualidad característicamente negativa u hostil, y se constituye en fuente de juicios valorativos distorsionados. En su descripción psicofenomenológica de la personalidad, Lersch (1971) incluye al resenti- miento como la expresión pasiva del afán vindicativo que surge cuando la expresión activa -la venganza- no es posible. El afán vindicativo, a su vez, surge cuando hay un daño o una insatisfacción de las necesidades vinculadas a las vivencias pulsionales del yo -conservación, egoísmo, poder, estima y autoestima. Buss (1996) lo define como un sentimiento de cólera a partir de un maltrato real o ficticio, verbalizado como disgusto, gemidos, queja y exigencia. Esta definición es interesante, ya que enfatiza dos ideas relevantes: la de maltrato, idea que coincide con el planteamiento de Lersch, y la de percepción subjetiva del mismo, lo que apunta a que una persona puede manifestar resentimiento a partir de su percepción subjetiva de haber sido maltratada, como también enfatiza Buela (2009).

Coincidiendo con estos planteamientos, Echeverría (1996) describe al resentimiento como uno de los cuatro estados de ánimo básicos, junto a la resignación, la aceptación y la ambición. Estos estados surgen como consecuencia de la actitud que la persona asume frente a los juicios que hace de su situación. De acuerdo a Echeverría, hay dos tipos de juicios posibles: de facticidad y de posibilidad. Un juicio de facticidad es la adscripción que hace la persona de inmodificabilidad de una situación. Es decir, una persona hace un juicio de facticidad cuando cree que alguna situación específica que la afecta es inmutable. Cuando se hace un juicio de esta naturaleza, la persona puede aceptarlo o rechazarlo; el resentimiento es el estado de ánimo resultante de rechazar una facticidad que es percibida como ataque $u$ ofensa hacia la propia persona. Así, el resentimiento se construye como estado emocional en la narrativa personal, en una conversación subyacente que representa la interpretación de haber sido víctima de un acto injusto por parte de otro u otros, frente al cual la persona se rebela. Lo injusto deviene de la idea de haber tenido derecho moral a obtener o hacer algo que, no obstante ese derecho, le fue negado (León, Romero, Novara \& Quesada, 1988); esta negación es la que es percibida como injusta, como un agravio hacia la persona. En esta interpretación, alguien aparece como culpable por impedir que se obtuviera lo que se merece; este alguien podría ser una persona, un grupo o una clase de personas, una institución, la sociedad, el mundo o la vida en general (Echeverría, 1996; Buss, 1996). 
Para Echeverría (1996), el resentimiento se nutre de las promesas y las expectativas incumplidas. Ambas confieren el derecho de esperar un determinado comportamiento de otros. Cuando alguien promete, confiere a la otra persona el derecho de esperar legítimamente que la promesa se cumpla. Sin embargo, hay ciertas convenciones sociales de comportamiento, propias de cada comunidad, que son independientes de las promesas que cada individuo formule explícitamente, y que generan legítimas expectativas. No tiene sentido, por ejemplo, afirmar: "Yo nunca prometí hacerme responsable de mis hijos", puesto que hay una convención social que hace innecesaria la formulación de dicha promesa para generar la expectativa de cumplimiento. Si las promesas o expectativas no se cumplen, y este incumplimiento es percibido como permanente, es altamente probable que surja el resentimiento, como una invocación de justicia frente a la injusticia del derecho no respetado. Como apuntan Buss (1996) y Echeverría (1996), esta percepción de injusticia puede tener sus bases en la ocurrencia de una ofensa o ataque manifiesto e intencional, o en la vivencia subjetiva de haber sido ofendido o atacado a partir de un acto carente de tal intencionalidad, pero al cual el sujeto resentido se la atribuye. Esto puede ocurrir especialmente cuando ya se ha experimentado resentimiento, ya que se comienza a percibir selectivamente aquellas situaciones que confirman la idea de haber sido víctima de acciones injustas, aumentando la posibilidad de atribuir esta intencionalidad a los actos de los demás. Por otra parte, generalmente no es un solo o único hecho el que gatilla el resentimiento; en la mayoría de los casos se trata de una acumulación de hechos experimentados subjetivamente como injustos. Así, puede resultar muy predisponente al resentimiento un ambiente en el cual a diario se presentan injusticias, postergaciones o maltratos (Castillo, 1982; León et al., 1988; Scheller, 1912/1938). De este modo, pequeños enojos o frustraciones adquieren importancia al acumularse, ya que por lo general tienen un efecto aditivo (Nathan \& Harris, 1989), favoreciéndose el resentimiento cuando se suceden situaciones de maltrato o postergación y/o cuando el sujeto va selectivamente otorgando mayor relevancia a aquellas que considera injustas (Titze, 1983, citado en León et al. 1988).
En cualquier caso, para que surja el resentimiento, la sola presencia de frustración o percepción de injusticia no es suficiente; es necesario que el impulso inmediato que surge para responder a la ofensa o ataque, o para reparar la injusticia, sea obstruído o imposible, ya que lo adecuado en tal caso sería la manifestación directa de tales sentimientos. El resentimiento es el resultado de esta coacción. Autores como Stack Sullivan (1964, citado en León et al., 1988) y Mueller (1983, citado en Troncoso, 1998) apuntan que el resentimiento puede surgir como resultado de la consideración que hace la persona acerca de las consecuencias que puede tener para él la expresión de sus sentimientos negativos hacia alguien que tiene una situación privilegiada de poder, o incluso, como resultado de amenazas violentas y represoras ejercidas por alguien, que hacen que la persona se sienta incapaz de defenderse. Cualquiera sea el origen de esta imposibilidad de manifestar los sentimientos negativos, el efecto es que la persona ejerce autocontrol evitando y posponiendo la expresión de la emoción negativa (Nathan \& Harris, 1989) y eventualmente desplazándola para otro momento y situación más apropiada; cuando esto se hace permanente, se transforma en resentimiento (Scheller, 1912/1938).

De este modo, el resentimiento es un estado de ánimo recurrente, que surge al ser o considerarse imposible la manifestación abierta de los sentimientos negativos hostiles surgidos por el rechazo de una situación o facticidad considerada como ofensiva o injusta, que produce daño o menoscabo a su persona o sus aspiraciones. Este estado de ánimo se expresa en disposiciones de acción indirectas o desplazadas. Así, tomando como base y ampliando la definición que hicieran León et al. (1988), asumimos como definición provisional de resentimiento la siguiente: estado de ánimo relativamente permanente que surge del juicio de haber sido maltratado o postergado injustamente en el logro de derechos o expectativas que se considera legítimos, que se manifiesta en actitudes hostiles hacia las cosas, las personas, la vida, el mundo o sí mismo. Operativamente, el resentimiento involucra: revivir constantemente sentimientos o emociones del pasado, relacionados con situaciones de abuso, postergación o injusticia, reales o imaginarias; autopercepción de ser víctima; creencia de haber obtenido menos de lo merecido; culpabili- 
zación de otros por su postergación; sentimiento de impotencia; retención o expresión indirecta de sentimientos negativos; agresividad latente; juicios críticos hacia el mundo externo; promesa y/o búsqueda de ocasiones que puedan dar lugar a actos de venganza internos; hostilidad.

Aunque algunas personas parecen más predispuestas que otras al resentimiento por razones personales (Scheller, 1912/1938), por nuestro ámbito de interés nos preocupan mucho más las razones sociales y las características del mundo moderno que contribuyen al mismo llevándolo desde el nivel personal al colectivo (Basabe \& Bobowik, 2013; Turner, 2011). Muchas de las situaciones características y frecuentes en nuestra sociedad que se constituyen en fuente de frustraciones y de incumplimiento de derechos y expectativas, devienen en gran parte de una característica esencial de la misma: su jerarquización, asociada a desigual distribución de recursos y poder. Como afirma Echeverría (1996), la relación entre distribución de poder y resentimiento es crítica. En una sociedad más igualitaria habría menos posibilidad de resentimiento, aunque, como advierte Scheller (1912/1938) la máxima carga de resentimiento corresponde a aquella sociedad en que los derechos políticos y la igualdad social están presentes a nivel discursivo, pero coexiste con diferencias muy notables en el poder, la riqueza y la educación efectivos. Éste es el caso de la mayor parte de nuestras sociedades latinoamericanas, que incorporan la igualdad en su discurso oficial y mediático, pero en las cuales las inequidades son también evidentes; de este modo, es fácil advertir cómo en nuestros sistemas de convivencia se presentan frecuente y sistemáticamente situaciones de atropello y abuso que pueden generar resentimiento, como el maltrato familiar, la discriminación étnica y social y las condiciones de pobreza. Los niños y jóvenes, en particular, se encuentran expuestos a situaciones de agravio social; el contexto escolar, por ejemplo, tiende a poner en desventaja a los niños y jóvenes de niveles socioeconómicos bajos (Castillo, 1982; Weinstein, 2001): el sistema educativo es contradictorio, elitista e injusto. La escuela quiere niños y jóvenes cuyas características poco corresponden a los niños y jóvenes pobres, en particular a aquellos que pertenecen al sector de la población que hoy en día se denomina vulnerable o vulnerada. Esta situación no puede sino producir un sentimiento de injusticia, que muchas veces, por temor a un mayor rechazo o simplemente por no percibir las desigualdades que le dan origen, no se orienta hacia las fuentes de la frustración, sino que comienzan a referirse a la vida, el mundo o la propia existencia (González, 1991; Troncoso, 1998; Zuhin, 2008). El autoritarismo, las descalificaciones constantes, las amenazas de castigo, hacen sentir al joven que no puede o no debe reaccionar frente a las fuentes de sus tensiones, acumulando sentimientos negativos que luego descarga en tiempos, espacios y personas que no se relacionan directamente con su problema (Castillo, 1982).

Interesados en el trabajo con jóvenes, particularmente en los ámbitos de la violencia escolar y de los jóvenes que presentan conductas desadaptativas o son infractores de ley, el resentimiento aparecía frecuentemente, por lo que hace más de una década nos propusimos buscar estrategias para abordarlo, conceptual y empíricamente. En ese empeño, dimos con el trabajo pionero de León et al. (1988) en que los autores daban cuenta de la construcción y validación de una escala para medir el resentimiento en jóvenes peruanos. No obstante, su definición no surgía de un modelo teórico explícito y el instrumento tenía una confiabilidad relativamente baja, probablemente por su reducida cantidad de ítems. Por otra parte, estaba orientada a una población universitaria, por lo que los ítems no resultaban apropiados para nuestra población de interés, principalmente jóvenes con escasa escolaridad en el caso de aquellos infractores de ley y de enseñanza media, en el caso del tema de la violencia escolar. Por esta razón, tomando el trabajo de León et al. como punto de partida, intentamos desarrollar un instrumento apropiado para estas poblaciones, coherente con un modelo teórico definido. El resultado permitió contar con un instrumento con el cual hemos desarrollado investigación, aunque el instrumento mismo y el proceso de su desarrollo no han sido reportados. Con este artículo se intenta saldar esa deuda, ya que la experiencia nos ha demostrado la utilidad del mismo para la investigación con jóvenes en condiciones de vulnerabilidad. En este trabajo se presenta, entonces, el proceso de desarrollo y validación del instrumento, que se llevó a cabo en dos estudios sucesivos, con el propósito de que los interesados en el tema 
puedan utilizarlo para establecer relaciones con otras variables o definir estrategias de acción respecto de grupos específicos.

\section{Estudio 1: Construcción y Validación Inicial del Instrumento}

Aunque el objetivo final era disponer de un instrumento válido para evaluar resentimiento en jóvenes con fines de investigación, coherente con una definición y un marco teórico explícito, el contexto en el que la necesidad surgió -la atención de menores vulnerados, vulnerables y eventualmente con dificultades adaptativas o incluso infractores de ley- nos exigía que también sirviera a un fin práctico, contribuyendo a describir y comprender las características de estos jóvenes. Con esta consideración en mente, se elaboró un conjunto de 150 afirmaciones que podrían reflejar resentimiento en jóvenes (tanto en sentido positivo -marcando la presencia de resentimiento- como negativo - marcando la ausencia de resentimiento) a partir del marco conceptual ya esbozado, incluyendo algunos de los cuales fueron tomados directamente de la escala de León et al. (1988).

Después de un exhaustivo examen, esta cantidad inicial quedó reducida a 129 afirmaciones, las que fueron presentadas a nueve jueces, junto con una definición ampliada de nuestro concepto de resentimiento. Se les solicitó que evaluaran cada ítem como esencial, útil pero no esencial, o innecesario, en relación a evaluar resentimiento en jóvenes. Siguiendo el criterio de Cohen y Cohen (1983), se mantuvieron sólo aquellos ítems que más de la mitad de los jueces consideró esencial, que fueron 62. A estos ítems se agregaron otros 12 que fueron sugeridos por los jueces, por lo que la escala quedó formada por 74 ítems con validez de contenido. Estos se presentan en el formato de una encuesta de actitudes hacia la vida, pidiéndose a los participantes que señalen si coinciden o no con cada afirmación, formato que fue aplicado en forma monitoreada a algunos jóvenes para detectar problemas de lectura o comprensión, lo que permitió mejorar algunos detalles de redacción y presentación.

En este primer trabajo, el objetivo principal fue evaluar la validez predictiva del instrumento resultante, a través de su capacidad para distinguir entre grupos definidos con alto y bajo nivel de resentimiento, aunque también se determinó preliminarmente algunas características psicométricas para su consideración en el estudio siguiente.

\section{Metodología}

\section{Participantes}

Para esta etapa inicial se escogió trabajar directamente con la población objetivo, por lo que se conformó una muestra intencionada -con y sin resentimiento, a juicio de expertos; el procedimiento se describe más adelante- $y$ de participación voluntaria, informada y consentida de 60 jóvenes infractores de ley y que, por esa razón, asistían a algún centro de atención de menores, sea en régimen de internado o ambulatorio. Los jóvenes tenían entre 13 y 19 años y pertenecían a ambos sexos, proporcionalmente.

\section{Instrumentos}

El instrumento principal fue la Escala de Actitud hacia la Vida, como se denominó al instrumento cuyo desarrollo se describió anteriormente. La mayor parte de los ítems puntúan en el sentido de presencia de resentimiento y unos pocos en sentido contrario. Para los primeros, el ítem puntúa cuando la respuesta es afirmativa, y para los segundos, cuando es negativa. Adicionalmente, se administró la escala de negación, una de las escalas de validez del Inventario de Comportamiento Interpersonal (ICl, Abarca \& Hidalgo, 1986), con el objeto de cautelar que el instrumento fuera válidamente respondido.

\section{Procedimiento}

Para conformar la muestra, se solicitó la colaboración de profesionales de centros de atención de menores de la ciudad de Arica, Chile. A quienes aceptaron colaborar, se les entregó un documento con la definición y las características centrales del resentimiento y se les pidió que, según dichos criterios y su experiencia con los jóvenes, identificaran algunos que, a su juicio, presentaran resentimiento y algunos que no lo hicieran. A partir de esta identificación, se invitó a jóvenes con y sin resentimiento a participar, conformándose dos grupos de 30 jóvenes que aceptaron colaborar, uno de los cuales estuvo integrado por quienes, a juicio de los profesiona- 
les, presentaban resentimiento, y uno por quienes no lo presentaban. A estos 60 jóvenes se les aplicaron el instrumento y luego se eliminaron 9 cuestionarios considerados inválidos por una de las siguientes razones: respuesta doble a uno o más ítems; menos de $80 \%$ de ítems respondidos; no alcanzar el criterio de validez en la escala de negación del ICI. Se contó así con un grupo clasificado como resentido, formado por 27 participantes, 10 mujeres y 17 hombres, y otro clasificado como no resentido, formado por 24 participantes, 15 mujeres y 9 hombres. Con los datos obtenidos sobre esta muestra, se realizó análisis de ítems tanto desde el punto de vista interno (relación ítem-total) como desde su capacidad de discriminar entre ambos grupos, y se reconfiguró la escala, para la cual se obtuvo la confiabilidad por consistencia interna. Finalmente se obtuvieron las puntuaciones en la escala reconfigurada para cada participante, se comparó los resultados de ambos grupos y se analizó el efecto de las variables sexo y edad, que se registraron como variables de control.

\section{Resultados}

En el análisis de ítems, se eliminó aquellos cuya relación con el puntaje total fue menor o igual a $r=.35$, quedando compuesta la escala por 36 ítems con buena relación ítem-total y que discriminan claramente entre los grupos clasificados como resentido y no resentido: al comparar ítem por ítem, el grupo no resentido puntúa por debajo del grupo clasificado como resentido en cada uno de ellos (Figura 1). La confiabilidad por consistencia interna para este conjunto de ítems fue de $\alpha=.92$.

La diferencia promedio en la puntuación total entre ambos grupos (Tabla 1$)$ fue de 11.73, altamente significativa $(t=6,32, \mathrm{gl}=$ $49, p<.0001)$. Respecto de las variables estructurales, se encontró que las mujeres puntuaron significativamente más que los varones $(t=2.605, g l=49, p=.012)$, pero que la variable edad no hacía diferencia $(F=.778, g l=6$, $p=.592$ ). Dada la influencia de la variable sexo, y como ambos grupos diferían en la proporción

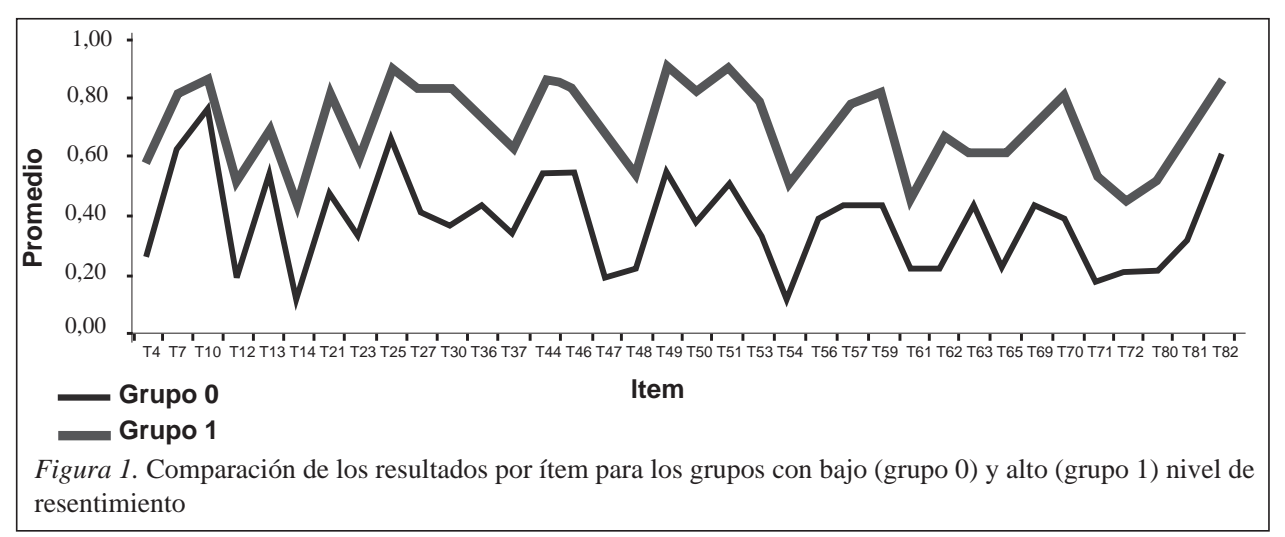

Tabla 1

Estadísticos descriptivos en la escala de actitud hacia la vida para los grupos no resentido y resentido, estudio 1

\begin{tabular}{lccccc}
\hline Grupo & $\begin{array}{c}\text { Número } \\
\text { de casos }\end{array}$ & Media & $\begin{array}{c}\text { Desviación } \\
\text { típica }\end{array}$ & Mínimo & Máximo \\
\hline $\begin{array}{l}\text { No } \\
\text { resentido }\end{array}$ & 27 & 13,85 & 6,649 & 2 & 24 \\
Resentido & 24 & 25,58 & 6,580 & 12 & 36 \\
\hline
\end{tabular}


de casos según esta variable, se los equiparó eliminando algunos casos al azar, y se repitió el análisis de la diferencia en el puntaje total entre los grupos resentido y no resentido, encontrándose que ella, aunque menor, mantiene su significación $(t=4.357, g l=36, p<.001)$.

\section{Discusión}

El análisis de los ítems de la Escala de Actitud hacia la Vida, mostró que la mayor parte de ellos resultaban adecuados, lo que permitió establecer criterios estrictos para su conservación en el instrumento, tanto desde el punto de vista de su consistencia interna como desde su capacidad para distinguir entre los participantes con y sin resentimiento. Por esta razón, no sorprende que la puntuación total muestre una buena capacidad para distinguir entre ambos grupos, incluso controlando la variable sexo, que hizo diferencias en la puntuación total. Esta diferencia por sexo es coherente con el marco propuesto, ya que, tratándose de un grupo integrado exclusivamente por jóvenes infractores de la ley, podría considerarse a las mujeres doblemente discriminadas. En primer lugar, por su condición de jóvenes (Dávila, 2009; Farías, 2011) y luego por su condición de mujeres, discriminación esta última que es transversal a toda la sociedad (Medel y Asún, 2014) y que se manifiesta incluso en el acceso a la justicia (Heredia, 2006). Adicionalmente, por la caracterización culturalmente estereotipada de las mujeres (Morales \& González, 2007), tienen menos posibilidades de responder a tales discriminaciones, por lo que serían más propensas a desarrollar resentimiento. Con estos resultados, se concluye que el conjunto de ítems seleccionados mide válidamente el resentimiento.

\section{Estudio 2: Forma Definitiva}

A pesar de los buenos indicadores obtenidos con esa forma del instrumento, tanto en la aplicación piloto como en una nueva revisión se obtuvo información que aconsejó introducir algunas mejoras; así, se eliminaron dos ítems cuyo contenido era redundante y se agregó otros 6 cuyo planteamiento es positivo, es decir, contrario a la presencia de resentimiento. Esto obedeció a dos criterios: primero, menguar al menos parcialmente el carácter mayoritariamente negativo de los ítems de la escala, y segundo, disminuir el posible efecto de aquiescencia, al proporcionar mayor cantidad de ítems que deberían ser respondidos en sentido inverso a los demás para mantener la consistencia. De esta manera, la escala definitiva quedó compuesta por 40 ítems, con la cual se llevó adelante un segundo estudio para determinar sus características psicométricas.

\section{Metodología}

\section{Participantes}

Participaron 575 jóvenes de entre 12 y 18 años con escolaridades entre el séptimo y el décimosegundo grados de escuelas de la ciudad de Arica, Chile, de los cuales la mitad fueron hombres y la mitad mujeres. El muestreo fue bietápico: en la primera etapa se realizó un muestreo intencionado de unidades completas (para determinar establecimientos educacionales y cursos dentro de los mismos), asegurando proporcionalidad por nivel socioeconómico; y en la segunda etapa, se realizó un muestreo aleatorio dentro de cada unidad completa seleccionada. De los jóvenes incluidos en la muestra resultante, el $70 \%$ asistía a escuelas públicas, el $26 \%$ a escuelas privadas subvencionadas y el $4 \%$ a centros de atención especializada a menores infractores de la ley.

\section{Instrumento}

La Escala de Actitud ante la Vida constó de 40 ítems de respuesta dicotómica, que presentan pensamientos, sentimientos y acciones concordantes con la presencia o ausencia de resentimiento, los que se puntúan de acuerdo a su concordancia o no con la presencia del mismo. Así, la puntuación puede variar entre 0 y 40 , en que 0 es la menor presencia de resentimiento y 40 la mayor.

\section{Procedimiento}

Los investigadores tomaron contacto con los establecimientos seleccionados y solicitaron autorización para contactar a los alumnos y aplicar el instrumento. Se invitó a participar a los estudiantes, incluyendo sólo a quienes aceptaron participar. A ellos se les administró la escala, en pequeños grupos o individualmente. Con los datos, se realizó análisis de ítems, se obtuvo la confiabilidad por consistencia interna 
(coeficiente alfa), se calculó el puntaje total para cada participante y se obtuvo los estadísticos descriptivos, que fueron comparados según las variables estructurales. Posteriormente, se realizó análisis factorial exploratorio, a fin de establecer la estructura factorial del instrumento.

\section{Resultados}

El análisis de ítems mostró que todos correlacionaban positivamente con la puntuación total de la escala, en un rango que varió entre $r=.128$ y $r=.504$. Desde el punto de vista de la consistencia interna no resultó razonable eliminar ninguno, ya que la confiablidad para el conjunto resultó de $\alpha=.84$, y todos contribuían a ella o, al menos, no la disminuían. La puntuación total se distribuyó en forma aproximadamente normal con $M=13.39$, y $D T=6.253 \mathrm{e}$ indicadores de asimetría y curtosis de 0.420 y - 0.274 respectivamente. Los estadísticos descriptivos para la escala total, considerando las variables estructurales, se muestran en la Tabla 2. Al analizar las diferencias entre los subgrupos definidos por las variables estructurales, no se encontró efecto significativo por sexo $(F=.011, g l=1, p=.917)$, pero sí tanto la edad como el curso, tomados separadamente, tuvieron un efecto altamente significativo $(F=2.123, g l=6, p<.05$ y $F=3.881$, $g l=6, p<.001$ respectivamente). Asimismo, hubo efecto significativo de la dependencia del establecimiento (público versus privado, $t=2.025, g l=573, p<.05)$ y la situación legal $(t$ $=2.586, g l=573, p<.01)$.

A pesar de que estos resultados permiten trabajar con el instrumento completo como una sola escala, sometimos los datos a análisis factorial exploratorio, a fin de mejor comprender la configuración del constructo. Aunque los métodos de análisis factorial usualmente utilizados han sido teóricamente objetados en el análisis de datos dicotómicos y se sugiere la utilización de métodos basados en el criterio de mínimos cuadrados generalizados, Ferrando (1996) señala que el análisis de componentes principales puede ser utilizado con bastante confianza como método de aproximación a pesar de su inadecuación teórica en ese caso, en particular cuando se cumple que los valores

Tabla 2

Estadísticos descriptivos para la puntuación total en la Escala de Actitud Ante la Vida, según variables estructurales, estudio 2

\begin{tabular}{|c|c|c|c|c|}
\hline Variable & Subgrupo & $n$ & $M$ & $D S$ \\
\hline \multirow[t]{2}{*}{ sexo } & Femenino & 287 & 12.7 & 6.00 \\
\hline & Masculino & 288 & 12.8 & 6.55 \\
\hline \multirow{7}{*}{ Edad (años) } & 12 & 44 & 12.1 & 7.03 \\
\hline & 13 & 98 & 12.3 & 5.41 \\
\hline & 14 & 98 & 12.9 & 6.03 \\
\hline & 15 & 104 & 14.1 & 6.19 \\
\hline & 16 & 93 & 13.0 & 6.24 \\
\hline & 17 & 87 & 13.3 & 7.04 \\
\hline & 18 & 51 & 10.6 & 6.05 \\
\hline \multirow{7}{*}{ Grado } & $6^{\circ}$ & 2 & 10.5 & 12.02 \\
\hline & $7^{\circ}$ & 120 & 12.5 & 6.17 \\
\hline & $8^{\circ}$ & 115 & 13.5 & 5.86 \\
\hline & $9^{\circ}$ & 105 & 13.8 & 7.00 \\
\hline & $10^{\circ}$ & 97 & 12.9 & 5.78 \\
\hline & $11^{\circ}$ & 80 & 13.3 & 6.47 \\
\hline & $12^{\circ}$ & 56 & 9.3 & 5.28 \\
\hline \multirow{2}{*}{$\begin{array}{l}\text { Dependencia del } \\
\text { establecimiento }\end{array}$} & Público & 428 & 13.1 & 6.44 \\
\hline & Privado & 147 & 11.9 & 5.69 \\
\hline \multirow[t]{2}{*}{ Situación legal } & No infractores & 550 & 12.7 & 6.24 \\
\hline & Infractores & 25 & 16.0 & 6.53 \\
\hline
\end{tabular}


estimados de comunalidad son altos y se tiene un número suficiente de variables; coincidiendo con Gorsuch (1974), señala que con más de 30 variables los resultados coinciden hasta el segundo decimal. En el caso de este conjunto de datos, la comunalidad promedio fue .509 con un valor mínimo de .367 , en 40 variables, por lo que se optó por seguir este procedimiento. Por otra parte, tanto la prueba de esfericidad de Bartlett $\left(X^{2}=4116,40, g l=780, p=0,000\right)$ como la medida de adecuación muestral de KaiserMeyer-Olkin (KMO $=0,827$ ) indican que los datos tienen un grado de intercorrelación que posibilita su análisis con técnicas factoriales (Pérez y Medrano, 2010).

Un primer análisis extrajo 11 componentes con autovalor mayor que 1 (Kaiser, 1960) que explicaban el 50,9\% de la varianza, pero el examen del gráfico de sedimentación (Catell, 1966, Figura 2) sugirió que soluciones de uno o tres factores podrían ser apropiadas. La solución de un factor fue descartada porque explicaba sólo el 14.7\% de la varianza y adicionalmente, no agrega información a lo que ya se conoce a través de la puntuación total porque todos los ítems cargan en el mismo, salvo que podría acortarse la cantidad de ítems descartando aquellos de menor carga.

Se optó, entonces, por forzar la solución a tres factores, la que explica el $27.7 \%$ de la varianza (Tabla 3). Las escalas que se desprenden de este análisis presentaron indicadores razonables de consistencia interna ( $\alpha=.749, \alpha$ $=.802$ y $\alpha=.612$, respectivamente) y resultaron claramente interpretables, por lo que se mantuvo esta solución.
El análisis factorial exploratorio aporta importante evidencia a favor de la validez del instrumento, y enriquece las posibilidades para su interpretación. Los dos primeros factores apuntan claramente al resentimiento, pero es posible distinguir con cierta claridad el matiz que los diferencia. El primero, caracterizado por el ítem "Creo que la vida ha sido más injusta conmigo", corresponde al núcleo central del resentimiento, el sentimiento de haber sido víctima de injusticia, e incluye ítems que reflejan emociones como rabia e impotencia frente a la misma. Por ello, hemos llamado a este factor, tentativamente, Reacción ante la Injusticia. El segundo factor, aunque también incluye algunos ítems cuyo contenido se aproxima a los del primer factor, enfatiza el ánimo revanchista, el tomar venganza o hacer pagar, como señala su ítem marcador, "Quiero vengarme de los que me causaron algún daño", por lo que le hemos denominado Afán Vindicativo. El tercer factor, marcado por el ítem "Me iento valorado por mis amigos", reúne todos los ítems positivos de la escala, que reflejan tanto una valoración propia como del mundo social inmediato, por lo que lo hemos llamado Valoración. A pesar de que la proporción de varianza explicada por estos tres factores es relativamente baja, las escalas que emergen del análisis resultan claramente coherentes con el constructo tal como lo hemos definido y muestran confiabilidades aceptables para su utilización. Esto posibilita que los resultados del instrumento sean utilizados como una sola puntuación o distinguiendo las subescalas configuradas a partir de este análisis.

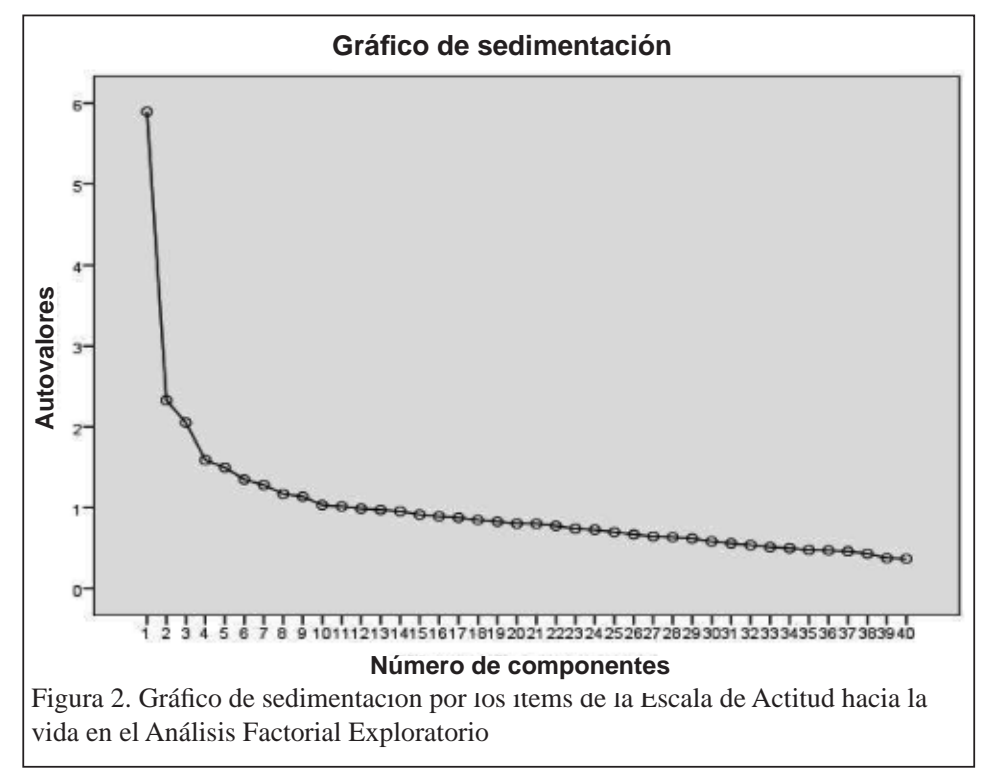


Tabla 3

Matriz de estructura de la solución de tres componentes

\begin{tabular}{|c|c|c|c|}
\hline \multirow{2}{*}{ Ítems } & \multicolumn{3}{|c|}{ Componente } \\
\hline & 1 & 2 & 3 \\
\hline Mis padres me ven como una persona con derechos iguales a los de ellos. & ,276 &,- 083 & ,293 \\
\hline Creo que el que ríe último ríe mejor. & ,038 &,- 268 &,- 037 \\
\hline Recuerdo con rabia a los que me culparon por cosas que no hice. & ,278 &,- 545 & ,132 \\
\hline Admiro a mis padres. &, 310 &,- 107 &, 503 \\
\hline Guardo rabia con quienes se han aprovechado de mí. &, 219 &,- 543 & 145 \\
\hline Me siento valorado por mis amigos. &, 071 &,- 135 & ,612 \\
\hline Me gusta hacer sufrir a mis padres porque se lo merecen. & ,451 &,- 139 & ,068 \\
\hline Si tengo la oportunidad, me desquito de las personas que me han tratado injustamente. & ,172 &,- 625 & ,007 \\
\hline Me molesta escuchar a los que hablan de lo bien que los trata la vida. & ,375 &,- 095 & ,106 \\
\hline He pateado cosas por no poder demostrar mi rabia. & ,299 &,- 169 & ,147 \\
\hline Me da rabia que en mi casa se fijen más en mis defectos. &, 267 &,- 206 & ,225 \\
\hline Me siento valorado cuando mis profesores reconocen mis logros. & 161 &,- 080 &, 579 \\
\hline Me hago daño cuando algo me da rabia. & ,440 &,- 154 & ,026 \\
\hline Quiero vengarme de los que me causaron algún daño. & ,220 &,- 724 & ,188 \\
\hline Sólo recuerdo lo bueno de mi familia, lo malo no importa. & 188 &,- 063 & ,323 \\
\hline Me enfurece que mis profesores me consideren menos que a otros. & ,168 &,- 216 & ,005 \\
\hline Guardo rencor contra quienes me han maltratado. & ,294 &,- 644 & , 154 \\
\hline Me agrada mucho cuando mis padres me expresan su afecto. & ,243 &,- 102 &, 521 \\
\hline Rompo cosas para reclamarle al mundo lo injustos que han sido conmigo. &, 549 &,- 274 & ,066 \\
\hline Guardo rabia contra mis padres por obligarme a hacer cosas que no quería. & ,489 &,- 261 &, 137 \\
\hline He deseado insultar a alguien por hacerme sentir inferior. & ,095 &,- 361 &, 117 \\
\hline Creo que la vida ha sido injusta conmigo. & ,603 &,- 198 & ,128 \\
\hline Me da rabia no poder expresar lo que siento en mi casa. & ,373 &,- 107 & ,229 \\
\hline Me siento valorado cuando mis amigos me defienden. &,- 021 &,- 061 &, 544 \\
\hline Quisiera que los que me despreciaron se arrepintieran y me pidieran perdón. & 137 &,- 263 &,- 218 \\
\hline Hacerme daño es una forma de hacer pagar a los demás. & ,417 &,- 181 &,- 006 \\
\hline Insultaría a alguien para desahogar la rabia que guardo. &, 270 &,- 405 & ,082 \\
\hline Me molesta que mi infancia haya sido menos feliz que la de otros. & ,492 &,- 216 &, 129 \\
\hline Algunos deberían pagar por haberme tratado mal. & ,289 &,- 639 & ,040 \\
\hline Siento agrado cuando le expreso cariño a los demás. & ,159 &,- 163 & ,373 \\
\hline Soy amable aún con los que me han causado algún daño. &, 094 &,- 434 & 153 \\
\hline Siento rencor contra mi padre o madre. & ,464 &,- 130 & ,289 \\
\hline Siento que soy marginado injustamente por las personas. & ,348 &,- 199 & ,238 \\
\hline Cuando alguien me trata mal, me las paga. & ,232 &,- 690 & ,053 \\
\hline Tengo entre ceja y ceja a quienes alguna vez se rieron de mí. & ,311 &,- 638 & 120 \\
\hline Mis amigos me quieren como soy. & ,022 &,- 114 &, 590 \\
\hline Cuando no puedo expresar mi rabia, me golpeo. &, 530 &,- 077 & ,085 \\
\hline $\begin{array}{l}\text { Una forma de vengarme de mis padres es hacerlos sentir culpables de lo que me } \\
\text { pasa. }\end{array}$ & ,486 &,- 221 &,- 015 \\
\hline Rompo cosas cuando recuerdo lo que otros me han hecho. &, 574 &,- 217 & ,083 \\
\hline Siempre recuerdo las veces que se me trató injustamente. & ,433 &,- 315 &, 157 \\
\hline
\end{tabular}

Nota. Se ha resaltado en negrita la carga de los ítems retenidos en cada componente. 


\section{Discusión General}

En este artículo intentamos proponer un instrumento que mida válidamente la presencia de resentimiento en jóvenes, tanto con fines de investigación -establecer relaciones con otras variables-, como para disponer de esa información como antecedente para diseñar intervenciones en contextos escolares, y particularmente, en el caso de jóvenes infractores de la ley; eventualmente, también para aportar información para la toma de decisiones por parte de quienes son responsables de definir su situación legal. En tal sentido, ambos estudios aportan evidencia que permite asumir que el instrumento propuesto resulta válido para tales objetivos, al marcar diferencias tanto entre jóvenes infractores respecto de no infractores, en el estudio dos, como entre los propios jóvenes infractores, distinguiendo válidamente aquellos

que presentan resentimiento o no de acuerdo al criterio de expertos con experiencia directa con los jóvenes evaluados.

El efecto de otras variables estructurales sobre la puntuación global, es menos concluyente. La influencia del sexo aparece relevante en el primer estudio pero no en el segundo; probablemente, esta diferencia responda a las diferentes características de ambas muestras, ya que la del primer estudio estuvo conformada sólo por jóvenes infractores. La influencia de edad y curso que aparece en el segundo grupo podría asociarse a características evolutivas que se acentúan en la adolescencia, lo que explicaría que el grupo intermedio en edad y curso aparezca con mayor propensión a mostrar resentimiento; sin embargo, es un hecho que merece más información empírica para su explicación.

La estructura factorial que se encuentra en el segundo estudio resulta muy coherente con la forma de conceptualizar el resentimiento, ya que recoge los elementos principales: el sentimiento de injusticia y el deseo incumplido y persistente de tomar revancha. Adicionalmente, y por la inclusión de ítems en sentido opuesto al resentimiento, se configura un tercer factor que también resulta muy coherente, pues aparece como la otra cara de la medalla, que es el reconocimiento y la valoración de sí mismo y los demás. Coincidentemente, otros autores (Diessner \& Lewis, 2007; Watkins, Woodward, Stone y Kolts, 2003) propusieron y validaron una escala de gratitud, que denominaron GRAT por sus siglas en inglés (Gratitude, Resentment and Appreciation Test).
Ellos operacionalizaron el constructo gratitud a partir de cuatro características: sentido de abundancia, apreciación de las cosas simples, aprecio de los demás y reconocimiento de la expresión de gratitud; pero sus resultados factoriales validaron sólo tres dimensiones (sentido de abundancia, aprecio de las cosas simples y aprecio de los demás), ya que las dos últimas características se confundieron en el mismo factor aprecio por los demás, que se corresponde bastante bien con el factor que hemos llamado Valoración en nuestra escala. Por otra parte, el primer factor del GRAT, correspondiente al sentido de abundancia, es medido en esa escala principalmente por ítems en sentido inverso, muy similares a los que cargan en nuestro primer factor, reacción ante la injusticia: el ítem con mayor carga en sentido de abundancia del GRAT es: "Por alguna razón, yo nunca tengo los descansos que los otros tienen", y el que le sigue es: "En mi vida me han pasado más cosas malas de las que merezco", cuyo sentido es prácticamente el mismo que el ítem con mayor carga en nuestro primer factor: "Creo que la vida ha sido injusta conmigo". De modo que ambas escalas parecen estar midiendo prácticamente lo mismo, aunque una lo hace desde la disposición a mirar la parte vacía del vaso y la otra desde la disposición a mirar la parte llena. Desde este punto de vista, parece acertada nuestra decisión de llamar a esta escala Escala de actitud ante la vida, ya que incluye ambas miradas.

A partir del procedimiento de construcción se espera haber logrado un instrumento válido, tanto desde el punto de vista del contenido -es decir, congruente con el constructo desarrolladocomo desde el punto de vista discriminante;es decir, desde su utilidad para diferenciar a personas con distinto grado de resentimiento 0 , como se ha discutido en el párrafo anterior, de gratitud o valoración, como hemos llamado al tercer factor de la escala. Por otra parte, como se habrá comprendido a partir de nuestra discusión del concepto mismo, el objetivo final no es caracterizar personas particulares, salvo si el reconocimiento de su estado de ánimo permite desarrollar instancias de apoyo más específicas, lo que habría que considerar con mucha prudencia; más bien, nuestro objetivo ha sido contar con un instrumento que nos permita dar cuenta de este estado de ánimo en cuanto ello puede ser útil para describir consecuencias de situaciones sociales, y para investigar fenómenos asociados a estas situaciones. 


\section{Referencias}

Abarca, N., \& Hidalgo, C. (1986). Traducción y adaptación del Inventario de Comportamiento Interpersonal de Manger y cols. (Memoria de Grado), Universidad Católica de Chile, Santiago, Chile.

Aggar, C., Ronaldson, S., \& Cameron, I. D. (2011). Selfesteem in carers of frail older people: Resentment predicts anxiety and depression. Aging \& Mental Health, 15(6), 671 - 678.

Basabe, N., \& Bobowik, M. (2013). Estatus grupal, discriminación y adaptación en inmigrantes latinoamericanos y africanos en España. Psicoperspectivas, 12(1), 5-29.

Buela, A. (2009). El resentimiento. Estudios: Filosofía, Historia, Letras, VII(90), 143 - 148.

Buss, A. (1996). Psicología de la agresión. BuenosAires: Troquel.

Castillo, C. (1982). Los niños del Perú, clases sociales, ideología y política. Lima: Desco.

Cattell, R. B. (1966). The scree test for the number of factors. Multivariate Behavioral Research, 1, 245 - 276.

Cohen, J., \& Cohen, P. (1983). Applied multiple regression/ correlation analysis for the behavior sciences. Nueva York: Hilldale Erlbaum.

Dávila, O. (2009). Jóvenes chilenos y sudamericanos: Demandas, aspiraciones y políticas de juventud. Ultima década, 17(31), 147 - 177.

Dalrymple, T. (1995). The uses of resentment. Psychology Today, 28(2), 30 - 31.

Delgado, H. (1938). Psicología general y psicopatología de las tendencias instintivas. Revista de Neuropsiquiatría, 1, 255 - 353.

Diessner, R., \& Lewis, G. (2007). Further Validation of the Gratitude, Resentment, and Appreciation Test (GRAT). The Journal of Social Psychology, 147(4), 445 - 447.

Echeverría, R. (1996). Ontología dellenguaje. Santiago: Dolmen.

Farías, M. (2011). Reflexiones en torno al impacto de programas de empleo para jóvenes en Argentina: Un estudio de caso. Ultima década, 19(35), 169 - 194.

Ferrando, P. J. (1996). Evaluación de la unidimensionalidad de los ítems mediante análisis factorial. Psicothema 8(2), 397-410. Recuperado de http://www.psicothema. com/pdf/38.pdf

Gorsuch, R. L. (1974) Factor analysis. Philadelphia: Saunders.

Feather, N. T., \& Nairn, K. (2005). Resentment, envy, schadenfreude, and sympathy: Effects of own and other's deserved or undeserved status. Australian Journal of Psychology, 57(2), 87 - 102.

Feather, N. T., \& Sherman, R. (2002). Envy, Resentment, Schadenfreude, and Sympathy: Reactions to Deserved and Undeserved Achievement and Subsequent Failure. Personality \& Social Psychology Bulletin, 28(7), $953-961$.

González, O. (1991). Muerte en el callejón: aportes a la comprensión del mundo psicológico del niño del tugurio. Infancia y violencia. Lima: CEDAPP.

Heredia , I. (2006). Es la violencia de género y el acceso a la justicia un asunto de Derechos Humanos. Revista Venezolana de Estudios de la Mujer, 11(26), 99 - 112.
Inga, J., \& Vara, A. (2006). Factores asociados a la satisfacción de vida de adultos mayores de 60 años en Lima-Perú. Universitas Psychologica, 5(3), 475 - 486.

Kaiser, H.F. (1960). The application of electronic computersto factor analysis. Educational and Psychological Measurement, 20, 141-151.

Knuckey, J. (2011). Racial Resentment and Vote Choice in the U. S. Presidential Election. Politics \& Policy, 39(4), 559 - 582.

León, R., Romero, C., Novara, J., \& Quesada, E. (1988) . Una escala para medir resentimiento. Revista latinoamericana de Psicología, 20(3), 331 - 354.

Lersch, P. (1971). La estructura de la personalidad. Barcelona: Scientia.

Lyons, G. C. B., Deane, F. P., Caputi, P., \& Kelly, P. J. (2011). Spirituality and the treatment of substancve use disorders: An esploration of forgiveness, resentment and purpose in life. Addiction Research \& Theory, 19(5), 459-469.

May, R. (1976). El hombre en busca de sí mismo. Buenos Aires: Central.

Medel, R., \& Asún, R. (2014) Encuestas de evaluación docente y sesgos de género: un estudio exploratorio. Calidad en la educación, 40, 171 - 199.

Morales, O. A., \& González, C. (2007). Consideraciones discursivas sobre el género en el discurso académico e institucional: ¿dónde está ella? Educere, 11(38), 433 - 453.

Nathan, P. E., \& Harris, S. L. (1989). Psicopatología y sociedad. México: Trillas.

Navarro, R. (2007). Emoción, Teoría Social y Estructura Social. Frónesis, 14(3), 121-125.

Nietzsche, F. (1887/1994). La genealogía de la moral. Madrid: M. E. Editores.

Pérez, E. R. y Medrano, L. (2010). Análisis factorial exploratorio: Bases conceptuales y metodológicas. Revista Argentina de Ciencias del Comportamiento, 2(1), 58 - 66

Perls, F. (1887/1993) Sueños y existencia. Santiago: Cuatro Vientos.

Rees, S., \& Silove, D. (2011). Sakit Hati: A state of chronic mental distress related to resentment and anger amongst West Papuan refugees exposed to persecution. Social Sciences \& Medicine, 73(1), 103 - 110.

Scheller, M. (1938). El resentimiento en la moral. Buenos Aires: Espasa-Calpe. (Originalmente publicado en 1912).

Troncoso, R. (1998). Estudio exploratorio sobre la violencia en jóvenes de enseñanza media (Memoria de Grado), Universidad de Tarapacá, Arica, Chile.

Turner, B. S. (2011). Max Weber and the spirit of resentment: The Nietzche legacy. Journal of Classical Sociology, 11(1),75-92.

Watkins, P. C., Woodward, K., Stone, T., \& Kolts, R. L. (2003). Gratitude and happiness: Development of a measure of gratitude, and relationships with subjective well-being Social Behavior \& Personality, 31(5), 431 -452.

Weinstein, J. (2001) Joven y alumno. Desafíos de la Enseñanza Media. Última Década, 15, 99 - 119.

Zembylas, M. (2010). Racialization/ethnicization of school emotional spaces: the politics of resentment. Race Ethnicity and Education, 13(2), 253-270.

Zuhin, A. (2008). A educação de Sísifo: sobre ressentimento, vingança e Amok entre professores e alunos. Educacao \& Sociedade, 29(103), 583-606.

Para citar este artículo:

Leal-Soto, F., Sepúlveda Muños, J., Vargas Gómez, S., Braniff Carrasco, M., \& Irrazábal Leiva,

M. (2015). Desarrollo de un instrumento para evaluación del resentimiento en jóvenes. Ciencias

Psicológicas 9(1): 43- 54 